\title{
Regeneración ósea guiada con implante unitario con nanosuperficie y betafosfato tricálcico
}

\section{Guided bone regeneration with nanosurface single-tooth implant and beta-tricalcium phosphate}

\author{
PATO MOURELO J* \\ JIMÉNEZ GUERRA A* \\ MONSALVE GUIL L* \\ SEGURA EGEA JJ** \\ VELASCO ORTEGA E***
}

\begin{abstract}
Pato Mourelo J, Jiménez Guerra A, Monsalve Guil L, Segura Egea JJ, Velasco Ortega E. Regeneración ósea guiada con implante unitario con nanosuperficie y betafosfato tricálcico. Av Periodon Implantol. 2010; 22, 3: 127-134.
\end{abstract}

\section{RESUMEN}

Introducción. El objetivo del presente caso clínico era mostrar los resultados del tratamiento con implantes dentales mediante regeneración ósea guiada.

Caso clínico. Un varón de 32 años es valorado para tratamiento implantológico del incisivo central superior derecho perdido. El plan de tratamiento comprende la eliminación de un quiste periapical residual, inserción de forma sumergida de un implante unitario con nanosuperficie Galimplant ${ }^{\circledR}$ y regeneración ósea guiada con membrana de colágeno reabsorbible BioGide ${ }^{\circledR}$ y betafosfato tricálcico KeraOs ${ }^{\circledR}$ como material de injerto. Después de 8 meses se realiza una segunda cirugía con colocación de un tornillo de cicatrización. Después de 2 semanas, se realiza la carga funcional del implante con una corona unitaria cementada. Después de un periodo de carga funcional de 12 meses, no ha habido complicaciones.

Conclusiones. Este caso clínico indica que el tratamiento con implantes dentales mediante la técnica de regeneración ósea guiada constituye una terapéutica implantológica con éxito.

PALABRAS CLAVE: Implantes orales, regeneración ósea guiada, biomateriales, betafosfato tricálcico, implantes con nanosuperficie, implantología oral.

\section{SUMMARY}

Introduction. The aim of this clinical case was to report the outcome of treatment with a dental implant by guided bone regeneration.

Clinical case. A 32-year man is diagnosed for implant treatment of the upper central right incisive loss. Treatment planning including elimination of residual periapycal cyst, submerged insertion of a Galimplant ${ }^{\circledR}$ nanosurface implant and guided bone regeneration with BioGide ${ }^{\circledR}$ resorbable collagen membrane and betatricalcium phosphate $\mathrm{KeraOs}^{\circledR}$ grafting. After a time period of 8 months, a second surgical stage were realized with the healing screw. After two weeks, implant were loaded with a cemented fixed single-crown. Not complications were observed after a 12 months follow-up.

Conclusions. This clinical case indicate that treatment with dental implant by guided bone regeneration constitute a successful treatment.

* Profesor Colaborador Docente de Odontología Integrada de Adultos. Facultad de Odontología. Profesor del Postgrado de Implantología Oral. Universidad de Sevilla.

** Profesor Titular de Patología y Terapéutica Dental. Facultad de Odontología. Profesor del Postgrado de Implantología Oral. Universidad de Sevilla.

*** Profesor Titular de Odontología Integrada de Adultos. Facultad de Odontología. Director del Postgrado de Implantología Oral. Universidad de Sevilla. 
KEYWORDS: Dental implants, guided bone regeneration, biomaterials, beta-tricalcium phosphate, nanosurface implants, implant dentistry.

Fecha de recepción: 3 de diciembre de 2009.

Aceptado para publicación: 15 de diciembre de 2009.

\section{INTRODUCCIÓN}

La atrofia o reabsorción del hueso alveolar relacionada con la pérdida dental por traumatismos, caries o periodontitis puede comprometer la inserción de los implantes dentales en una posición prostodóncica ideal. En este sentido, el aumento de un volumen óseo insuficiente, es frecuentemente realizado de forma conjunta a la inserción del implante para conseguir unos buenos resultados funcionales y estéticos a largo plazo (1-3).

Después de una exodoncia en el maxilar superior se desarrolla un proceso de remodelación alveolar con una progresiva atrofia ósea en sentido vertical y vestíbulo-palatina que se incrementa con la acción de la prótesis removible. La cicatrización del alvéolo y su progresiva regeneración ósea suele durar entre 4-6 semanas, aunque la remodelación definitiva puede llegar hasta los 4 meses $(4,5)$. Desde un punto de vista morfológico, a los 6-12 meses, existe una reducción de 5-7 mm en sentido horizontal o vestíbulo lingual, lo que representa casi el 50\% de la anchura alveolar inicial. A estos cambios horizontales se acompañan modificaciones en la altura o apicoronales con una reducción de 2 a $4,5 \mathrm{~mm}(6,7)$. Para prevenir estas alteraciones en la disponibilidad ósea de la cresta alveolar se ha desarrollado la técnica de los implantes postextracción que consiguen mantener un buen nivel de volumen óseo $(8,9)$.

La reabsorción progresiva del hueso alveolar después de la exodoncia puede limitar la disponibilidad ósea en sentido horizontal para la inserción del implante. Si el soporte óseo es inadecuado, puede ser necesario la utilización de técnicas quirúrgicas complementarias para incrementar la anchura ósea como puede ser los expansores u osteótomos que aprovechando el módulo de elasticidad del hueso maxilar permiten con éxito la inserción de los implantes (10-12).

Las técnicas de regeneración tisular con utilización de una membrana y biomaterial de relleno pueden obte- ner una mejor cicatrización ósea y reducir la pérdida ósea crestal en el tratamiento con implantes dentales (13). De hecho, esta técnica de regeneración ósea puede ser recomendada, sobre todo, cuando se han producido dehiscencias o pérdida de alguna pared ósea durante la extracción que pudiera comprometer la inserción del implante, así como cuando se utiliza en zonas con presencia de infección o lesiones periapicales quísticas (14).

Las técnicas de aumento del volumen óseo para la inserción de implantes requieren, frecuentemente, la utilización de materiales de injertos como pueden ser el propio hueso del paciente o substitutos óseos (1-3). Entre los sustitutos óseos o biomateriales se encuentran los de origen animal (ej. hueso bovino o porcino mineralizado) o sintéticos (ej. hidroxiapatita, sulfato cálcico) (1-3). El betafosfato tricálcico (B-TCP) es un material sintético con propiedades osteoconductoras que permite por sus características biológicas y fisicoquímicas mantener bien el espacio rellenado habiendo sido utilizado con éxito en implantología oral (15-17).

Desde un punto de vista clínico, un aspecto esencial en las situaciones implantológicas complejas con compromiso óseo es la importancia del diseño macro y microscópico del implante (18). La morfología roscada del implante proporciona el diseño necesario para conseguir una excelente estabilidad primaria que asegure la cicatrización del lecho implantario; mientras que la superficie microrrugosa del implante es recomendable para conseguir un alto grado de oseointegración (19-21).

La incorporación de la nanotecnología a la investigación implantológica ha posibilitado el desarrollo de nuevas superficies de implantes que están incrementado la respuesta ósea y mejorando el grado de oseointegración $(22,23)$. Los resultados con implantes con nanotopografía parece indicar una excelente respuesta clínica en el tratamiento de los pacientes con edentulismo parcial y total (24). Además, estas nuevas nano- 
superficies pueden mejorar la unión del implante al hueso de baja densidad como el área posterior del maxilar superior (25).

El objetivo del presente estudio es valorar la eficacia clínica de los implantes con nanosuperficie en defectos óseos regenerados con betafosfato tricálcico.

\section{CASO CLÍNICO}

LTR. Paciente de sexo masculino, de 32 años de edad, sin antecedentes médicos de interés, que acude a consulta para tratamiento implantológico por pérdida del incisivo central superior derecho. El examen radiológico (ortopantomografía) muestra un quiste radicular residual a nivel del diente perdido. Al paciente se le recomienda la posibilidad de realizar un plan de tratamiento que consiste en una misma sesión operatoria, de la eliminación del quiste, regeneración ósea del defecto óseo e inserción de un implante. Posteriormente después de un periodo de cicatrización se realizará la rehabilitación con una corona cementada implantosoportada. El paciente es informado de la técnica quirúrgica y de los aspectos prostodóncicos (prótesis provisional y definitiva), temporalización (duración del tratamiento, número de visitas) y el seguimiento (revisiones clínicas y radiológicas), y de la posibilidad de la existencia de complicaciones y pérdida de implantes. El paciente autoriza el tratamiento implantológico mediante un consentimiento informado.

\section{Cirugía}

El día previo a la cirugía, el paciente comienza un régimen antibiótico preventivo (amoxicilina + clavulánico) durante una semana, y en el caso de existir dolor o inflamación, se le prescribe ibuprofeno. Además se le recomienda un enjuague diario con clorhexidina durante los primeros 30 días. Se realiza la extirpación del quiste radicular con legrado y se realiza el lecho implantario, insertándose un implante de conexión externa universal con nanosuperficie Nanoblast ${ }^{\circledR}$ (Galimplant, Sarria, España) de forma sumergida. Se rellena todo el defecto óseo con betafosfato tricálcico KeraOs $^{\circledR}$ (Keramat, Coruña, España) y se cubre con una membrana de colágeno reabsorbible BioGide ${ }^{\circledR}$ (Geistlich, Wolhusen, Suiza) (Figs. 1-6). A los 8 meses después de la inserción del implante se realiza la segunda cirugía con colocación de un tornillo de cicatrización (Figs. 7 y 8 ).

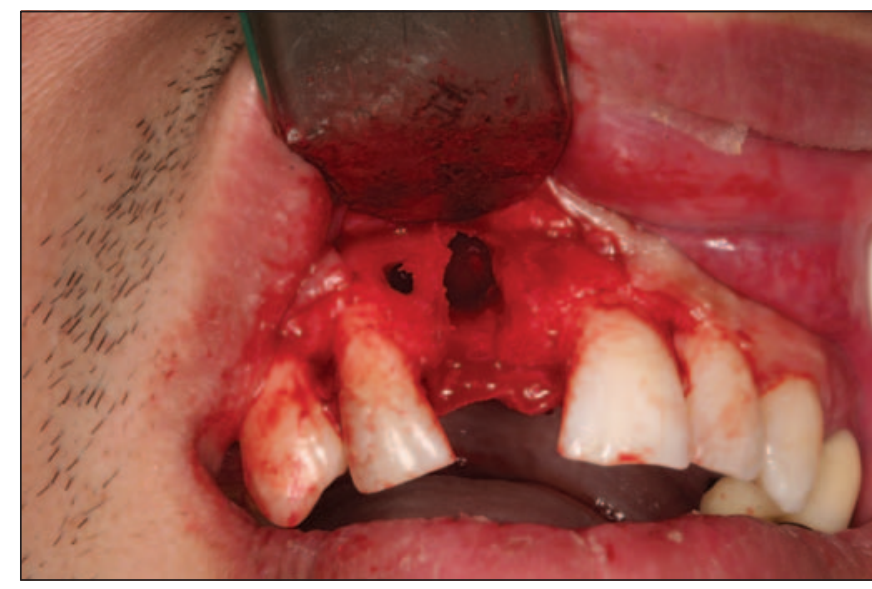

Fig. 1: Aspecto clínico del paciente con pérdida del incisivo central superior derecho y presencia de quiste periapical residual.

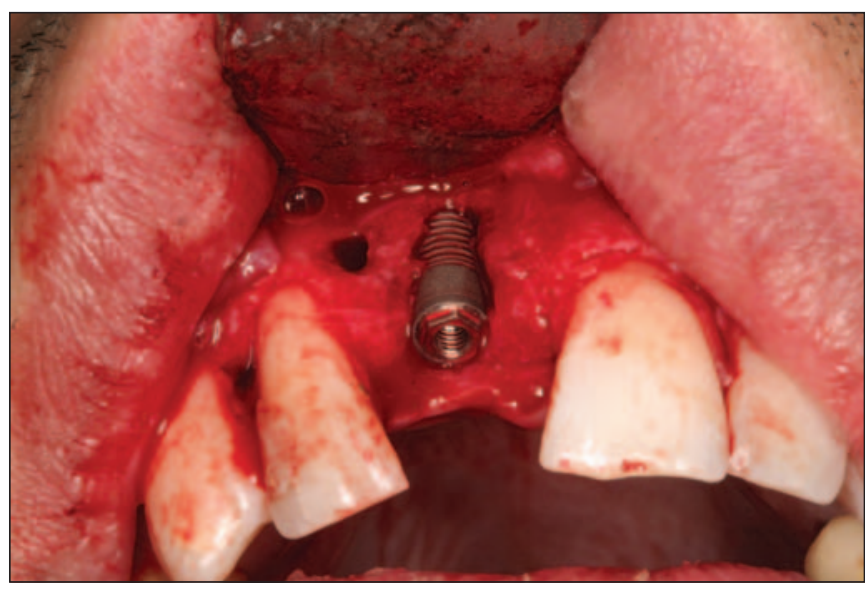

Fig. 2: Implante de conexión externa universal Galimplant ${ }^{\circledR}$ insertado.

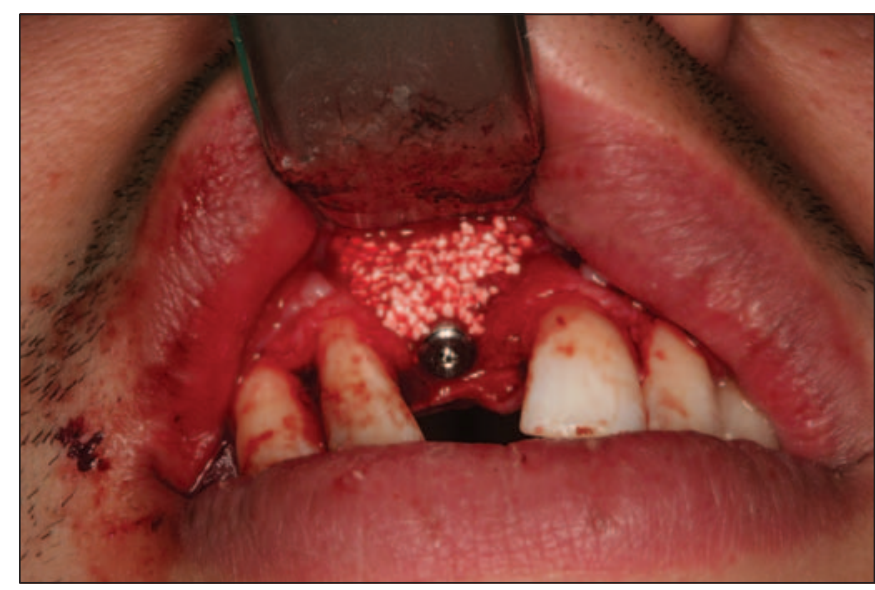

Fig. 3: Relleno del defecto óseo con betafosfato tricálcico Keraos ${ }^{\circledR}$. 


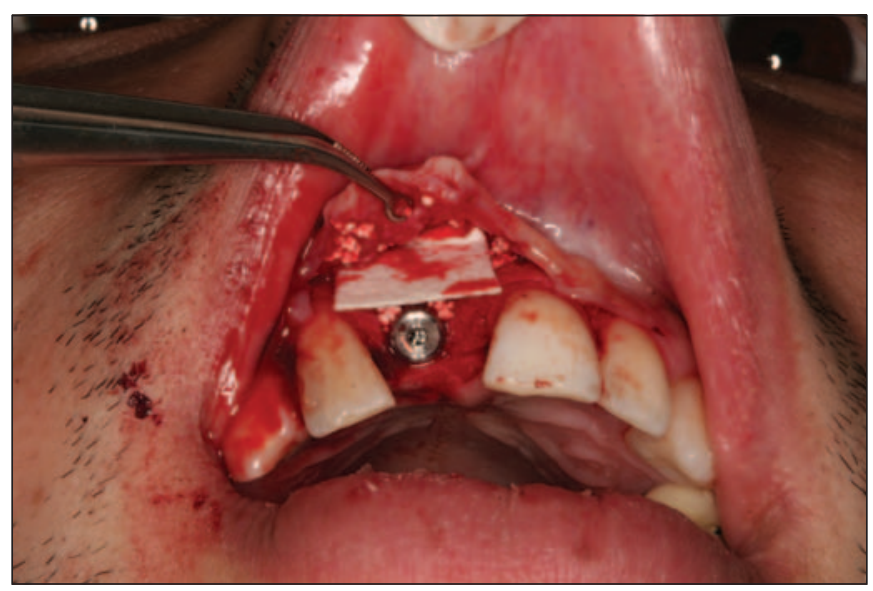

Fig. 4: Colocación de membrana reabsorbible de colágeno BioGide ${ }^{\circledR}$.

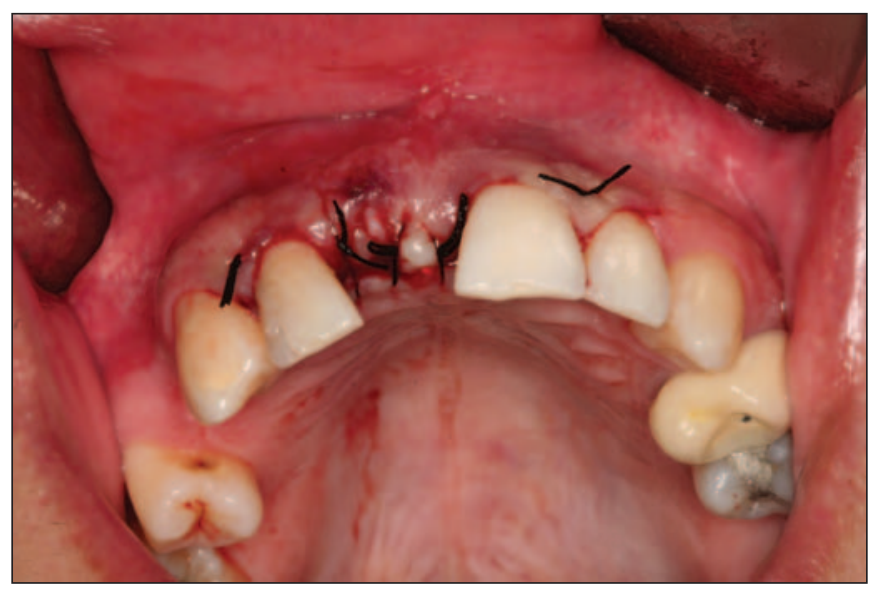

Fig. 5: Sutura después de la cirugía implantológica con regeneración tisular guiada.

\section{Prostodoncia}

A las 2 semanas de la segunda cirugía, se realizó la carga funcional del implante mediante la colocación de la correspondiente prótesis implantosoportada, una corona de porcelana cementada sobre un pilar metálico hexagonal mecanizado (Figs. 9 y 10 ).

\section{Seguimiento clínico}

El tiempo transcurrido de seguimiento clínico desde la carga funcional del implante ha sido de 12 meses. En este periodo de tiempo no ha habido complicaciones.

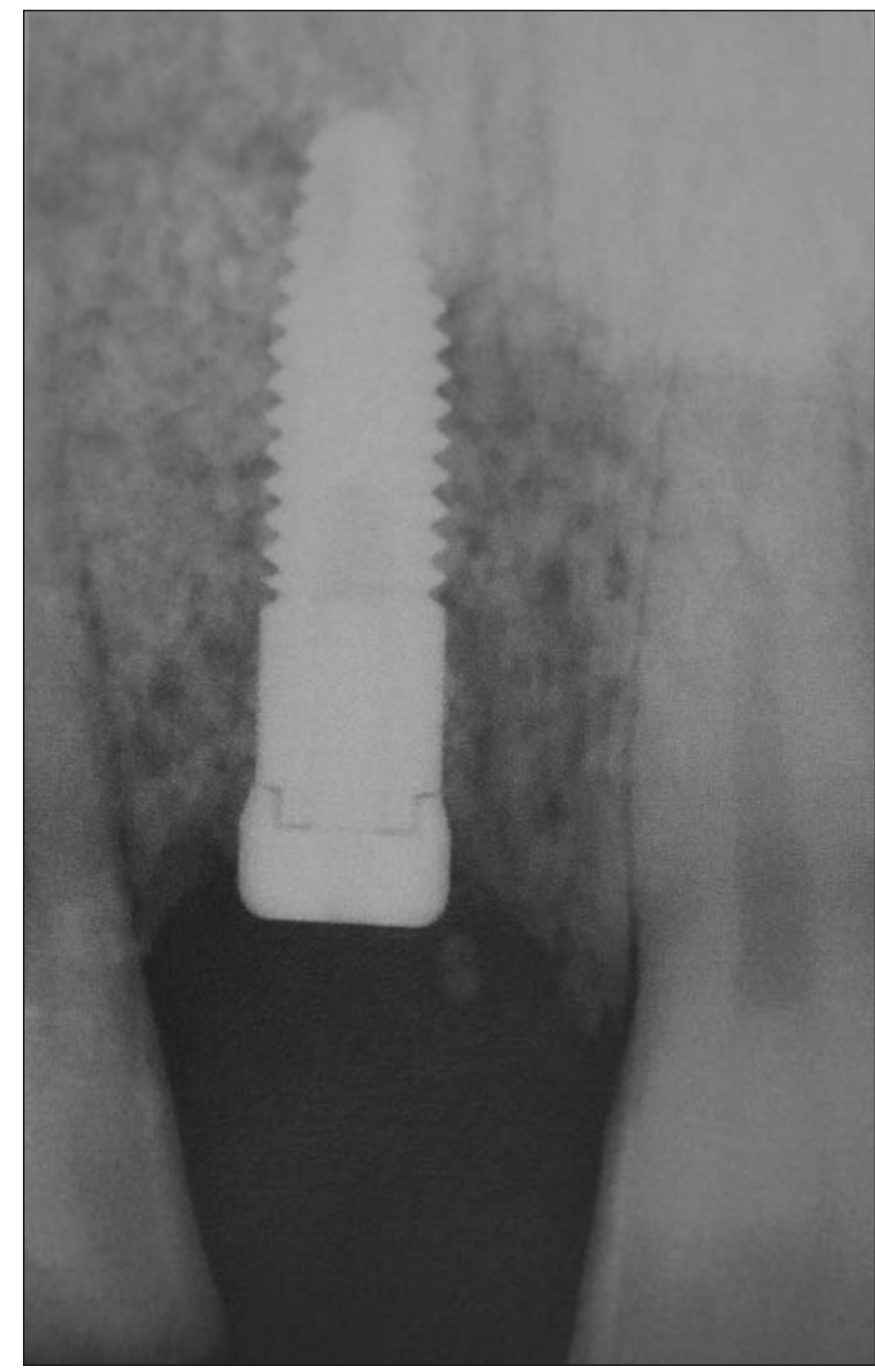

Fig. 6: Imagen radiográfica del implante insertado con el biomaterial injertado.

\section{DISCUSIÓN}

La regeneración ósea guiada constituye una técnica que fue desarrollada en periodoncia para el tratamiento de los defectos óseos periodontales. Su filosofía biológica consistía (después de la eliminación cuidadosa del cálculo subgingival y tejido inflamatorio) en facilitar el crecimiento y regeneración del tejido óseo, separando a través de una membrana, el hueso alveolar periodontal de los tejidos blandos superiores (encía, mucosa y periostio) $(26,27)$. De esta forma se evitaba la invasión de la zona que va a ser regenerada por las células del epitelio gingival y del tejido conjuntivo y se favorecía el crecimiento de las células progenitoras del ligamento periodontal en la superficie radicular $(26,27)$. 


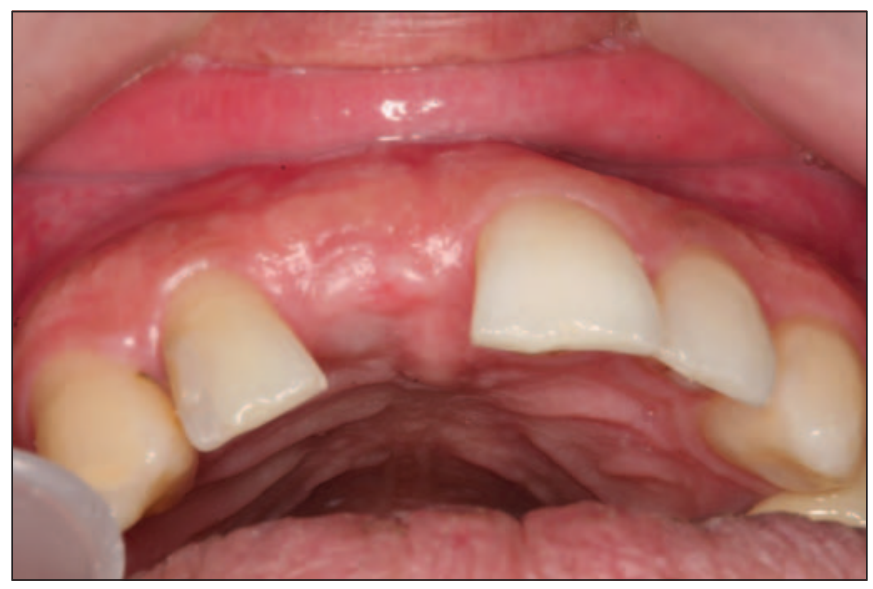

Fig. 7: Aspecto clínico a los 8 meses de la cirugía.

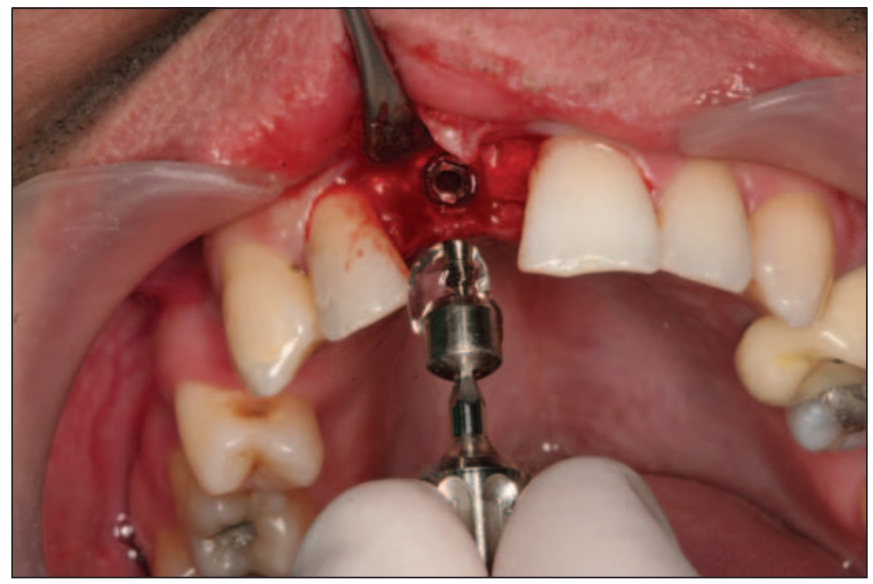

Fig. 8: Segunda cirugía. Colocación del tornillo de cicatrización.

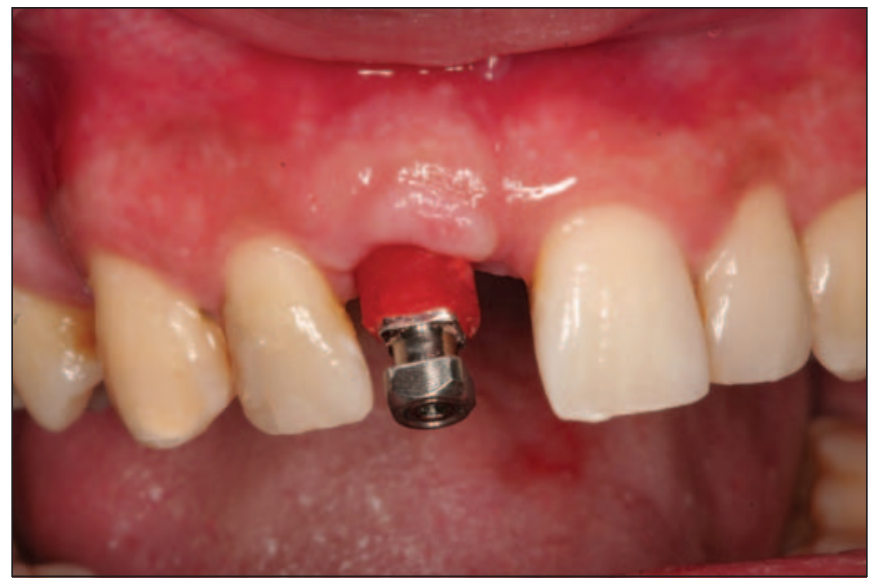

Fig. 9: Toma de impresiones para la realización de la prótesis unitaria correspondiente.

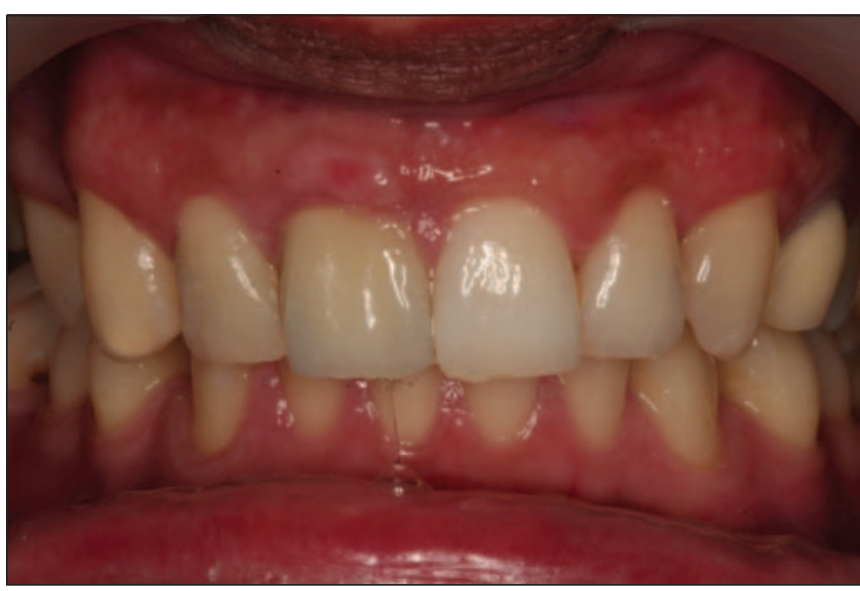

Fig. 10: Aspecto clínico de la corona unitaria cementada.

Posteriormente, la regeneración ósea guiada se ha ido incorporando a la práctica implantológica con buenos resultados clínicos para de forma, frecuentemente, simultánea a la inserción de los implantes, favorecer la cicatrización ósea e incrementar el volumen óseo que asegure la estabilidad primaria del implante y su oseointegración (28-30).

De hecho, la colocación de una membrana reabsorbible es recomendable porque ayuda a mantener el espacio ocupado por el biomaterial y lo estabiliza, facilitando la regeneración ósea, evitando una segunda cirugía ya que no necesita su retirada o eliminación. Además las membranas de colágeno, como la utilizada en el presente caso clínico, presentan un manejo relativamente fácil y tienen una excelente capacidad hemostática (27-31).

Además, la utilización de membranas reabsorbibles unida a un biomaterial de relleno parece mejorar los resultados clínicos comparados con la utilización solamente de membranas, como demuestra un estudio donde la posición final del margen de los tejidos blandos en relación al cuello del implante era significativamente más alto entre los pacientes tratados con membranas y material de relleno (32).

En el presente caso clínico se ha utilizado como biomaterial, el betafosfato tricálcico que ha demostrado una gran eficacia clínica en el tratamiento implantológico, especialmente como material de relleno en defectos óseos, periodontales y en implantología oral, especialmente en la cirugía de elevación del seno maxilar (15-17). 
El betafosfato tricálcico puede constituir un buen biomaterial para rellenar y sellar aquellos defectos óseos originados por la reabsorción del hueso alveolar, preservando el reborde alveolar y favoreciendo un volumen de hueso suficiente para la inserción del implante. Para que tenga lugar una buena regeneración ósea del defecto óseo, en el presente caso clínico se trataba de un quiste residual, es necesario la eliminación cuidadosa de la patología periapical, realizando un legrado profundo óseo y retirando el tejido inflamatorio de granulación, la fibrina y otros residuos orgánicos (33). Posteriormente durante la realización del lecho implantario puede ser recomendable la obtención de hueso del propio paciente para ser mezclado con el betafosfato tricálcico y aportar las células precursoras de osteoblastos y células madre mesenquimales que con las plaquetas (factores de crecimiento) pueden favorecer la osteogénesis y la osteoinducción respectivamente en el tejido injertado (33).

En el presente caso clínico, después de un periodo de cicatrización de 8 meses, al paciente se le realizó la segunda cirugía implantológica con la colocación del tornillo de cicatrización para 2 semanas más tarde realizarle la carga funcional del implante con una corona cementada. En este sentido, diversos estudios han demostrado que entre los 6 y 12 meses, el betafosfato tricálcico presenta una buena estabilidad biológica y física conjunta (33-36). Desde un punto de vista biológico a los 6 meses se ha demostrado que las células progenitoras óseas han migrado entre los poros del biomaterial favoreciendo la regeneración y sustitución por tejido óseo, además a los 6 meses los bordes del biomaterial se están reemplazando por hueso neoformado (34). Desde un punto de vista físico, el betafosfato tricálcico mantiene bien el espacio rellenado y proporciona un tejido duro y estable que asegura la estabilidad primaria de los implantes $(35,36)$.

Aunque la técnica de regeneración tisular guiada puede constituir un factor de riesgo para el éxito del tratamiento con implantes, porque supone su inserción sobre un tejido formado por hueso y un biomaterial injertado recubierto por una membrana que debe estimular la regeneración ósea, sin embargo la experiencia clínica demuestra que es posible obtener una elevada tasa de éxito en el resultado a largo plazo de los implantes con esta técnica implantológica, ya que la revisión de la literatura presenta resultados superiores al 95\% (2-10).

En el presente caso clínico, la cirugía implantológica y la regeneración ósea guiada fue realizada en un paciente que presentaba un quiste residual periapical que puede incrementar el riesgo de fracaso (37). Sin embargo, existen estudios que demuestran que no hay diferencias en el éxito de los implantes cuando se insertan en localizaciones con y sin patología periapi$\mathrm{cal}(38)$.

La calidad del hueso alveolar es un factor importante para conseguir la oseointegración. La situación clínica se complica cuando además, existe un volumen óseo insuficiente y existe una patología periapical residual, como sucede en el presente caso clínico, donde para insertar el implante es necesario la técnica de regeneración ósea guiada con utilización de biomateriales (betafosfato tricálcico) y membrana reabsorbible de colágeno. En estos casos más complejos, la superficie del implante es importante porque puede favorecer la oseointegración en un tejido con baja densidad e injertado con material de relleno $(39,40)$.

En este sentido, la estructura superficial nanométrica de los implantes dentales, como la utilizada en el presente caso clínico, puede incrementar la estabilidad primaria del implante y el contacto hueso-implante y favorecer la cicatrización precoz en áreas de baja densidad ósea (25-39). Los resultados a 18 meses de seguimiento clínico medio demuestran que los implantes con nanosuperficie presentan una tasa elevada de éxito (98\%) como demuestra un estudio con $200 \mathrm{im}$ plantes en 58 pacientes rehabilitados con coronas unitarias (43,5\%); puentes fijos $(28,2 \%)$; rehabilitaciones completas fijas (18\%) y sobredentaduras $(10,3 \%)(24)$.

\section{CONCLUSIONES}

La regeneración tisular guiada con la utilización de biomateriales como el betafosfato tricálcico y membrana reabsorbible de colágeno con inserción de implante con nanosuperficie puede constituir un tratamiento con buenos resultados clínicos para mantener el volumen y la calidad ósea necesarios el éxito de la rehabilitación protésica de pérdidas dentales unitarias.

\section{BIBLIOGRAFÍA}

1. Esposito M, Grusovin MG, Coulthard P, Worthington HV. The efficacy of various bone augmentation procedures for dental implants: a Cochrane systematic review of randomized controlled clinical trials. Int J Oral Maxillofac Implants 2006;21:696-710. 
2. Donos N, Mardas N, Chadha V. Clinical outcomes of implants following lateral bone augmentation: systematic assessment of available options (barrier membranes, bone grafts, split osteotomy). J Clin Periodontol 2008;35 (suppl. 8):173-202.

3. Jensen SS, Terheyden H. Bone augmentation procedures in localized defects in the alveolar ridge: clinical results with different bone grafts and bone-substitute materials. Int J Oral Maxillofac Implants 2009;24 (suppl): 218-36.

4. Amler MH. The time sequence of tissue regeneration in human extraction wounds. Oral Surg Oral Med Oral Pathol 1969;273:309-18.

5. Evian CI, Rosenberg ES, Cosslet JG, Corn H. The osteogenic activity of bone removed from healing extraction sockets in human. J Periodontol 1982;53: 81-5.

6. Johnson $\mathrm{K}$. A study of the dimensional changes occurring in the maxilla following tooth extraction. Aust Dent J 1969;14:241-4.

7. Lam RV. Contour changes of the alveolar processes following extractions. J Prosthet Dent 1960;10:25-32.

8. Lazzara RM. Immediate implant placement into extraction sites: surgical and restorative advantages. Int J Periodontics Restorative Dent 1989;9:333-43.

9. Velasco E, Pato J, Lorrio JM, Cruz JM, Poyato M. El tratamiento con implantes dentales postextracción. Av Perio Impl Oral 2007;19 (Supl.):35-42.

10. Chiapasco M, Zaniboni M, Boisco M. Augmentation procedures for the rehabilitation of deficient edentulous ridges with oral implants. Clin Oral Impl Res 2006;17 (Suppl. 2):136-59.

11. Shalabi MM, Manders P, Mulder J, Jansen JA, Creughers A. A meta-analysis of clinical studies to estimate the 4.5year survival rate of implants placed osteotome technique. Int J Oral Maxillofac Implants 2007;22:1 10-6.

12. Velasco E, Pérez O, Pato J, Lorrio JM, Cruz JM. La expansión ósea en la implantología oral. Av Perio Impl Oral 2008;20:95-101.

13. Zitzmann NU, Schärer P, Marinello CP. Long-term results of implant treated with guided bone regeneration: a 5year prospective study. Int J Oral Maxillofac Implants 2001;16:355-66.
14. Fiorellini JP, Nevins ML. Localized ridge augmentation/ preservation. A systematic review. Ann Periodontol 2003;8:321-7.

15. Zijderveld SA, Zerbo IR, van der Bergh JPA, Schulten EAJM, ten Bruggenkate CM. Maxillary sinus floor augmentation using a beta-tricalcium phosphate (Cerasorb) alone compared to autogenous bone grafts. Int J Oral Maxillofac Implants 2005;20:432-40.

16. Ormianer Z, Palti A, Shifman A. Survival of immediately loaded dental implants in deficient alveolar bone sites augmented with beta-tricalcium phosphate. Implant Dent 2006;15:395-403.

17. Velasco E, Pato J, Pérez O, Medel R, Segura JJ. La utilización del betafosfato tricálcico como biomaterial en implantología oral. Av Perio Impl Oral 2007;19:141-9.

18. Tetè S, Mastrangelo F, Traini T, Vinci R, Sammartino G, Marenzi G, Gherlone E. A macro-and nanostructure evaluation of a novel dental implant. Implant Dent 2008;17:309-20.

19. Shalabi MM, Gortemarker A, Van't Hof MA, Jansen JA, Creugers NHJ. Implant surface roughness and bone healing: a systematic review. J Dent Res 2006;85:496500 .

20. Misch CE, Wang HL, Misch CM, Sharawy M, Lemons J, Judy KWM. Rationale for the application of immediate load in implant dentistry: Part II. Implant Dent 2004; 13:310-21.

21. Martinez H, Davarpanah M, Missika P, Celleti R, Lazzara R. Optimal implant stabilization in low density bone. Clin Oral Impl Res 2001;12:423-32.

22. Le Guehennec L, Martin F, López-Heredia MA, Lourarn G, Amouriq Y, Cousty J, Layrolle P. Osteoblastic cell behavior on nanostructured metal implants. Nanomed 2008;3:61-71.

23. Meirelles L, Curie F, Jacobson M, Albrektsson T, Wennerberg $A$. The effect of chemical and nanotopographical modifications on the early stages of osseointegration. Int J Oral Maxillofac Implants 2008;23: 641-7.

24. Velasco E, Pato J, Jiménez A, Pérez O, Monsalve L, Segura JJ. La experiencia clínica con implantes dentales con nanosuperficie. Un estudio prospectivo a los 18 meses. Rev Esp Odontoestomatol Impl. En prensa. 
25. Orsini G, Piatelli M, Scarano A, Petrone G, Kenaly J, Piatelli A, Caputi S. Randomized, controlled histologic and histomorphometric evaluation of implants with nanometer-scale calcium phosphate added to the dual acid-etched surface in the human posterior maxilla. J Periodontol 2007;78:209-18.

26. Gottlow J, Nyman S, Lindhe J, Karring T, Wennströn J. New attachment formation in the human periodontium by guided tissue regeneration. J Clin Periodontol 1986;13: 604-16.

27. Sculean A, Nikolidakis D, Schwarz F. Regeneration of periodontal tissues: combinations of barrier membranes and grafting materials-biological foundation and preclinical evidence. J Clin Periodontol 2008;35 (suppl. 8):106-16.

28. Buser D, Dula K, Hirt HP, Schenk RK. Lateral ridge augmentation using autografts and barrier membranes: a clinical study with 40 partially edentulous patients. J Oral Maxillofac Surg 1996; 54:420-32.

29. Hämmerle CHF. Membranes and bone substitutes in guided bone regeneration. En: Lang NP, Karring T, Lindhe J (Eds.). Proceedings of the 3rd European Workshop on Periodontology. Implant Dentistry Quintessence: Berlin 1999;pag:468-99.

30. Blanco J, Alonso A, Sanz M. Long-term results and survival rate of implants treated with guided bone regeneration: a 5-year case series prospective study. Clin Oral Impl Res 2005;16:294-301.

31. Misch CE, Suzuki JB. Tooth extraction, socket grafting, and barrier membrane bone regeneration. En: Misch CE. Comteporary Implant Dentistry. San Luis: Elsevier Mosby 2009;pag:870-904.

32. Cornelini R, Cangini F, Martuscelli G, Wennström J. Deproteinized bovine bone and biodegradable barrier membranes to support healing following immediate placement of transmucosal implants: a short-term controlled clinical trial. Int J Perio Rest Dent 2004;24:555-63.

33. Palti $A$, Hoch T. A concept for the treatment of various dental bone defects. Implant Dent 2002; 1 1:73-8.

34. Zerbo IR, Bronckers ALJJ, Lange G, Burger EH. Localisation of osteogenic and osteoclastic cells in porous b-tricalcium phosphate particles used for human maxillary sinus floor elevation. Biomaterials 2005;26: 1445-51.

35. Suba Z, Takács D, Matusovits D, Barabás J, Fazekas A, Szabó G. Maxillary sinus floor grafting with betatricalcium phosphate in humans: desnity and microarchitecture of the newly formed bone. Clin Oral Impl Res 2006; 17:102-8.

36. Velasco E, Pato J, Garcia A, Medel R, López J. Estudio clínico e histológico del betafosfato tricálcico en la elevación del seno maxilar. Av Perio Impl Oral 2008;20: 147-54.

37. Chen ST, Buser D. Clinical and esthetic outcomes of implants placed in postextraccion sites. Int J Oral Maxillofac Implants 2009;24 (suppl):186-217.

38. Siegenthaler DW, Jung RE, Holderegger C, Roos M, Hammerle $\mathrm{CH}$. Replacement of teeth exhibiting periapical pathology by immediate implants. A prospective, controlled clinical trial. Clin Oral Impl Res 2007; 18: 727 37.

39. Velasco E, Pato J, Segura JJ, Medel R, Poyato M, Lorrio $\mathrm{JM}$. La investigación experimental y la experiencia clínica de las superficies de los implantes dentales. Parte I. Dentum 2009;9:101-7.

40. Velasco E, Pato J, Segura JJ, López J, Garcia A, España A. La investigación experimental y la experiencia clínica de las superficies de los implantes dentales. Parte II. Dentum 2009;9:108-13.

41. Goené RJ, Testori T, Trisi P. Influence of a nanometerscale surface enhancement on de novo bone formation on titanium implants: a histomorphometric study in human maxillae. Int J Periodontics Restorative Dent 2007;27:211-9.

\section{CORRESPONDENCIA}

Prof. Eugenio Velasco Ortega

Facultad de Odontología de Sevilla.

Avicena, s/n

41009 Sevilla

e-mail: evelasco@us.es 